\title{
1 Enhanced visuomotor learning and generalization in expert surgeons
}

2 Christopher L. Hewitson ${ }^{1,2}$, David M. Kaplan ${ }^{1,2^{*}}$

3

$4 \quad{ }^{1}$ Department of Cognitive Science, Macquarie University, Sydney, Australia

$5 \quad{ }^{2}$ Perception and Action Research Centre, Macquarie University, Sydney, Australia

6

$7 \quad{ }^{*}$ Corresponding Author

$8 \quad$ E-mail: david.kaplan@mq.edu.au

9

10 Both authors conceived and designed the experiments. C.L.H performed the

11 experiments and analyzed the data. Both authors wrote the paper and edited the

12 manuscript.

13

14 The authors declare no competing interests.

15

16

17 Short title: Visuomotor learning in expert surgeons 


\section{Abstract}

Although human motor learning has been intensively studied for many decades, it remains unknown whether group differences are present in expert cohorts that must routinely cope with and learn new visuomotor mappings such as minimally invasive

22 surgeons. Here we show that expert surgeons exhibit greater adaptation and 23 generalization compared to naive controls in a standard visuomotor adaptation task.

24 These findings run counter to a widespread background assumption in the field of motor

25 learning that visuomotor adaptation performance should be largely uniform across the

26 adult human population. Our findings also indicate that differences in basic visuomotor

27 learning capacities, either innate or acquired, might be an important source of difficulty in

28 learning and performing minimally invasive surgery. This information holds potential to

29 guide surgical candidate selection or optimize training programs to address individual 30 needs.

\section{Introduction}

33 Human motor learning has been intensively studied for many decades [1, 2]. However,

34 insufficient attention has been given to individual- or group-level differences in visuomotor

35 learning. For example, it remains unknown whether group differences are present in 36 expert cohorts that must routinely cope with and learn new visuomotor mappings such as 37 minimally invasive surgeons.

Laparoscopic or minimally invasive surgery (MIS) is rapidly replacing traditional open surgery for many procedures due to its major benefits for patients over conventional

40 open surgery including reductions in infection risks, recovery times, scarring, and overall 
41 hospital stays [3]. Despite these advantages, the task environment in MIS places high

42 demands on surgeons, increasing the difficulty relative to open surgery for both initial

43 learning [4] and ongoing performance [3,5,6]. Since laparoscopic instruments are

44 controlled through small insertion points in the skin, instrument movements are often

45 mirror-reversed and counter-intuitive (e.g., leftward hand motion produces rightward

46 instrument tip motion, and vice versa). Because surgeons receive visual feedback

47 indirectly via a laparoscopic camera that is in turn projected to a video display, rather than

48 through direct observation, they must also contend with a range of visualization problems

49 including absent depth information, variable magnification, and a restricted and frequently

50 distorted (e.g., rotated) field of view. These factors, which are often subsumed under the

51 general rubric of "challenges for hand-eye coordination" [7], also impose heavy

52 computational demands on the brain and likely contribute to the significant increase in

53 time to achieve proficiency in MIS compared to open surgery [8]; [9]

54 A related and potentially deeper explanation for why MIS is difficult to learn is that

55 it requires complex sensorimotor transformations [10, 11]. Sensorimotor transformations

56 involve the conversion of sensory inputs into appropriate motor commands [12], and are

57 known to introduce errors even for simple goal-directed movements such as pointing to

58 a visible target $[13,14,15]$. Moreover, because this sensory-to-motor mapping can and

59 often does change during MIS such as when the laparoscopic camera rotates relative to

60 the workspace or the fulcrum point of the instrument shifts, the same motor commands

61 will not always lead to the same outcome. Consequently, surgeons must be particularly

62 adept at learning new visuomotor mappings or transformations so they can maintain

63 accurate motor performance during a procedure despite these changes. To appreciate 
64 the inherent challenges involved, one can imagine trying to use a computer mouse if the

65 mapping between mouse and cursor movement changed frequently and unpredictably.

66 Therefore, if MIS introduces challenges for learning appropriate sensorimotor

67 transformations, this predicts that expert surgeons who have successfully learned to

68 overcome these challenges, will perform better than naive controls in a standard

69 visuomotor adaptation task $[16,17]$. This is precisely the hypothesis we sought to test in

70 this study.

71 Specifically, we predicted that expert minimally invasive surgeons would adapt

72 more rapidly and more completely and would subsequently generalize their learning more

73 widely than naive controls in a standard visuomotor adaptation task $[17,18]$. We found

74 that expert surgeons both adapted faster and more completely and generalized to a

75 greater extent across a range of novel target directions compared to controls. This study

76 provides valuable insights into the improved visuomotor learning capacities among

77 experts who draw on a vast reservoir of experience learning new visuomotor

78 transformations. It provides evidence for the existence of group-level differences in

79 visuomotor adaptation. Finally, it indicates that a standard visuomotor rotation task may

80 have predictive validity for learning rates in training tasks specifically designed for MIS

$81[19]$.

82

\section{Materials and Methods}

\section{Participants}

8510 expert surgeons and 10 naive controls participated in the study. All were right-hand

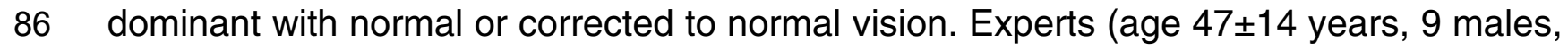


871 female) had all completed greater than 100 procedures (mean $\pm S D=2900 \pm 6000$; range

$88=>150<22000)$. Naïve controls $(23 \pm 3$ years, 4 males, 6 females $)$ were university

89 undergraduates with no prior medical experience, surgical training, or regular video game

90 use ( $\leq 3$ hours per week) [20-22]. All participants gave written informed consent to

91 participate and the experimental protocols were approved by the [redacted for review]

92 Human Research Ethics Committee (approval \#5201833993962).

94 Experimental Apparatus

95 A unimanual KINARM endpoint robot (BKIN Technologies, Kingston, Ontario, Canada) 96 was utilized in the experiments for motion tracking and stimulus presentation. The

97 KINARM has a single graspable manipulandum that permits unrestricted 2D arm

98 movement in the horizontal plane. A projection-mirror system enables presentation of 99 visual stimuli that appear in this same plane. Subjects received visual feedback about 100 their hand position via a cursor (solid white circle, $2.5 \mathrm{~mm}$ diameter) controlled in real101 time by moving the manipulandum. Mirror placement and an opaque apron attached just 102 below the subject's chin ensured that visual feedback from the real hand was not available 103 for the duration of the experiment.

\section{Experimental Procedure}

106 Subjects were instructed to perform fast and accurate reaching movements with the 107 dominant (right) arm using cursor feedback, whenever it was available. Subjects 108 performed reaches from a start target located at the center of the workspace to 11 109 different target locations $9 \mathrm{~cm}$ away from the start target and spaced $30^{\circ}$ apart (Fig 1) The 
110 start target was a solid red circle (5 mm diameter), and each reach target was a solid

111 green circle (5 $\mathrm{mm}$ diameter). The appearance of the reach target served as the go cue.

112 Subjects were positioned so that the starting target was directly in front of their torso.

113 Although these data are not reported here, a second workspace was also tested. In this

114 experiment, the target array was translated rightward, which required subjects to extend

115 their shoulder joint clockwise $45^{\circ}$ to acquire the start target [23].

117 Fig 1. Experimental paradigm. (a) During the baseline block, subjects performed 118 reaches to 1 of 11 targets with full visual feedback for $2 / 3$ of the trials and no visual 119 feedback for $1 / 3$ of the trials, (b) During the adaptation block, subjects performed reaches 120 to 1 target with visual feedback rotated at the endpoint of the movement, (c) During the 121 generalization block, subjects performed reaches to 1 of 11 target (10 untrained target 122 directions) without visual feedback, (d) Target array used in the experiment.

124 The experiment began with a familiarization block of 33 reach trials (3 per target in 125 pseudorandom order) with veridical visual feedback provided throughout the reach. After 126 the familiarization phase, subjects rested for 1 minute. The baseline block consisted of 127198 reach trials (18 per target). For two-thirds of the reaches (132 trials), veridical cursor 128 feedback was provided throughout the trial. For one-third (66 trials), visual feedback was 129 withheld. During no-feedback trials, the cursor disappeared as soon as it left the start 130 target. Visual feedback reappeared after the handle was positioned within a $1 \mathrm{~cm}$ 131 diameter from the center of the start target. After the baseline phase, subjects rested for 1321 minute. The adaptation block consisted of 110 reaches toward a single target positioned 
133 at $0^{\circ}$ in the frontal plane. As the subject reached toward the target, cursor feedback was

134 rotated about the start target by $30^{\circ}$ (CW or CCW; counterbalanced between subjects).

135 For the cursor to move directly toward the target, hand motion would need to be directed

$13630^{\circ}$ opposite to the direction of the cursor rotation. For all reaches, no visual feedback

137 was provided for the duration of the reach. For $90 \%$ of the trials, visual feedback was

138 provided at the end of the movement for $150 \mathrm{~ms}$. For the remaining $10 \%$ of the trials, no

139 visual feedback was provided during the reach or at the endpoint. The generalization

140 block consisted of 66 reaches across 11 novel target directions (6 trials per direction) in

141 pseudorandom order. Visual feedback was withheld for the entire outward reach

142 movement and was only provided when the subject's hand came within $1 \mathrm{~cm}$ of the start

143 target during the return movement.

145 Data Analysis and Models

146 Movement kinematics including hand position and velocity were recorded for all trials

147 using BKIN's Dexterit-E experimental control and data acquisition software (BKIN

148 Technologies). Data was recorded at $200 \mathrm{~Hz}$ and logged in Dexterit-E. Custom scripts for

149 data processing were written in MATLAB and data analysis was performed in JASP

150 (0.9.2). A combined spatial- and velocity-based criterion was used to determine

151 movement onset, movement offset, and corresponding reach endpoints [24, 25, 26].

152 Movement onset was defined as the first point in time at which the movement exceeded

$1535 \%$ of peak velocity after leaving the start target. Movement offset was similarly defined

154 as the first point in time at which the movement dropped below $5 \%$ of peak velocity after

155 a minimum reach of $9 \mathrm{~cm}$ from the start target in any radial direction. Reach endpoints 
156 were defined as the $x$ and $y$ values at movement offset. Reaction time (RT), total

157 movement time (MT), peak velocity (PV), and endpoint error (EE; cursor position at

158 movement offset - target position) were calculated for every trial across all experimental

159 blocks and compared between groups and conditions via analysis of variance (ANOVA)

160 and Welch T-tests. Learning rate was determined by fitting a power function to the data:

$f(x)=a x^{b}$

164 Because this assumes that learning is monotonic, which may not always be the case in 165 visuomotor adaptation [27], data were also analyzed using repeated-measures ANOVA and Welch T-tests. Greenhouse-Geisser corrected values for ANOVAs are reported in case sphericity was violated. Generalization of learning to new target directions was compared between experts and controls using repeated-measures ANOVA and was quantified further by fitting subject data to a simple model in which generalization, $g(\theta)$, decreases as a Gaussian function of distance from the trained target direction [28, 23]:

$172 \quad g(\theta)=k \cdot e^{\frac{-\left(\theta-\theta_{0}\right)^{2}}{2 \sigma^{2}}}$

174 where $\theta$ is the trained target direction, $\theta_{0}$ is the peak or center of the generalization 175 function (i.e., the target direction showing maximal adaptation), $k$ is the magnitude or size 176 of the peak (i.e., the amount of adaptation expressed in the maximal direction, and $\sigma$ is 177 the width. Goodness-of-fit was evaluated by calculating the coefficient of determination ( $\left.178 r^{2}\right)$ 
Results

181 To assess performance differences between groups, we measured reaction time (RT), total movement time (MT), peak velocity (PV), and endpoint error (EE; cursor position at

183 movement offset - target position) for every trial across all experimental blocks. We began

184 by investigating differences in reach performance between expert surgeons and controls 185 prior to adaptation. During baseline reaches in which no visuomotor perturbation was 186 imposed, experts and novices differed across a wide range movement parameters. 187 Experts were more accurate (smaller endpoint error) and more precise (less variable) 188 than controls $\left(E E=1.0 \pm 2.3^{\circ}(S D)\right.$ to the left of the target averaged across all target 189 directions compared to $E E=3.9 \pm 5.7^{\circ}$ to the left for controls; $p<.001$, Welch unpaired $t-$ 190 test) (Fig. 2). We also compared reaction time (RT = movement onset - go cue onset). 191 Experts showed significantly $(p<.001)$ faster reaction times $(R T=350 \pm 160 \mathrm{~ms})$ than 192 controls $(R T=460 \pm 90 \mathrm{~ms})$. Next, we compared total movement time (MT = movement 193 offset - movement onset). Experts were significantly $(p<.001)$ quicker to complete reaches 194 (MT $=770 \pm 140 \mathrm{~ms}$ ) compared to controls $(\mathrm{MT}=901 \pm 200 \mathrm{~ms})$. Interestingly, peak velocity 195 was significantly $(p<.001)$ greater for controls $(P V=15.0 \pm 0.7 \mathrm{~cm} / \mathrm{s})$ than for experts (PV $196=14.0 \pm 0.6 \mathrm{~cm} / \mathrm{s})$.

198 Fig 2. Baseline reaches for all target directions. (a) Representative expert, (b) 199 Representative control. 
201 Since this analysis collapsed across target directions and feedback conditions, we also 202 performed ANOVAs for the within-subject factors of TARGET (11 target directions) and

203 CONDITION (feedback vs no-feedback) and the between-subject factor of GROUP 204 (experts vs controls) for all movement parameters. While there was a significant effect of 205 GROUP, no GROUP x CONDITION x TARGET interactions were observed indicating no 206 differences across target directions or feedback conditions.

207 Table 1. Baseline reach data for experts and controls.

\begin{tabular}{|l|c|c|c|c|}
\cline { 2 - 5 } \multicolumn{1}{c|}{} & $\begin{array}{c}\text { Endpoint error } \\
\left(^{\circ}\right)\end{array}$ & $\begin{array}{c}\text { Reaction time } \\
(\mathrm{ms})\end{array}$ & $\begin{array}{c}\text { Movement time } \\
(\mathrm{ms})\end{array}$ & Peak velocity \\
\hline Experts & $1.0 \pm 2.3^{\circ}$ & $350 \pm 160$ & $770 \pm 140$ & $14.0 \pm 0.6$ \\
\hline Controls & $3.9 \pm 5.7$ & $460 \pm 190$ & $910 \pm 200$ & $15.0 \pm 0.7$ \\
\hline
\end{tabular}

209 Next, we tested whether performance during and after adaptation differed between 210 experts and controls. We were specifically interested in whether there were group 211 differences in the learning rate, which can be defined as the proportion of endpoint error 212 that is corrected for in the subsequent movement. First, learning rates were estimated by 213 fitting a decaying power function to the data (see Methods). Fitted values indicate that 214 experts learned more rapidly than controls during the adaptation phase ( $\alpha=31.26$, vs $\alpha$ $215=26.48 .8$ respectively [Fig 3]). However, the coefficients of determination were relatively 216 low $\left(r^{2}=0.472, r^{2}=0.377\right.$, respectively) so we also performed parametric comparisons.

217 The within-subject factor of BIN and the between-subject factor of GROUP were 218 compared via repeated-measures ANOVAs across 11 bins of 10 trials per bin. There was 
219 a significant within-subject effect of $\operatorname{BIN}\left(F_{1,7.465}=59.6, p<.001, \omega^{2}=0.231\right.$; Greenhouse-

220 Geisser corrected) as well as a significant between subject effect of GROUP $\left(F_{1,7.465}=\right.$

$221269, p<.001, \omega^{2}=0.573 ;$ Greenhouse-Geisser corrected). These results indicate that

222 while both groups adapted rapidly, experts did in fact adapt significantly faster than

223 controls (Fig 3). In addition, averaged over the last two bins of trials during the adaptation

224 block, experts adapted to the perturbation more completely than controls $(29.6 \pm 2.0$

225 [98.7\%] compared to $28.5 \pm 4.3^{\circ}$ [94.9\%], $\mathrm{p}<.001$; respectively, baseline adjusted).

226 Reaction time and duration decreased over the course of the adaptation phase while peak

227 velocity increased for both experts and controls (Fig 3). Although not our primary focus,

228 there were significant within-subject effects of BIN as well as significant between subject

229 effects of GROUP for all remaining movement parameters (Table 1).

230

231 Fig 3. Learning rates for experts and controls. Green indicates experts and blue

232 indicates controls. Thin solid lines show group means. Shaded region indicates s.e.m.

233 Thick solid lines show model fits.

235 Finally, we investigated whether generalization of learning differed between the groups.

236 Local generalization to new, untrained target directions decreased as a function of

237 distance from the trained direction (up to $\pm 90^{\circ}$ from the trained target direction) for both

238 experts and controls (Fig 4). The within-subject factor of TARGET and the between-

239 subject factor of GROUP were compared via repeated-measures ANOVAs across the 11

240 targets with 6 trials per target. There was a significant within-subject effect of TARGET

$241\left(\mathrm{~F}_{1,8.674}=2518.2, \mathrm{p}<.001, \boldsymbol{\omega}^{2}=0.950 ;\right.$ Greenhouse-Geisser corrected $)$ as well as a 
242 significant between-subject effect of GROUP $\left(F_{1,8.674}=12276, p<.001, \omega^{2}=0.990\right)$. These

243 results indicate that generalization varies with target direction, and that experts generalize

244 significantly differently compared to controls.

Figure 4. Generalization patterns for experts and controls. Green indicates experts

247 and blue indicates controls. Error bars indicate s.e.m. Dashed lines indicate Gaussian

248 model fits.

250 To further quantify the difference in generalization between experts and controls, endpoint 251 data from targets were averaged across subjects and fit to a simple Gaussian model (see

252 Methods) with four free parameters: center location, magnitude, width and vertical offset

$253[23,28]$. The fitted values for the magnitude and width parameters indicate that experts

254 adapted to a far greater extent than controls ( $k=97.1 \%$ vs $k=70.1 \%)$ and generalized

255 their learning more broadly $\left(\sigma=43.9^{\circ}\right.$, vs $\left.\sigma=31.1^{\circ}\right)$ than controls $\left(r^{2}=0.94, r^{2}=0.96\right.$,

256 respectively). Expressing generalization as a percentage of the actual adaptation amount

257 rather than complete adaptation (100\%) had little effect (98.4\% vs $73.7 \%)$, since both

258 groups almost completely adapted to the imposed visuomotor rotation by the end of the 259 adaptation block.

261 Discussion

262 In the current study, we found that expert minimally invasive surgeons exhibit enhanced

263 visuomotor learning in every measure we tested. Specifically, experts adapted to the 264 imposed visuomotor rotation more rapidly and more completely than controls. They also 
265 generalized their learning to new target directions more broadly and to a considerably 266 greater extent.

What might explain these different learning patterns? One possibility is that

268 attentional differences between surgeons and controls account for the observed

269 differences in visuomotor learning performance. For example, there is some indication

270 that extensive video game use can enhance visual selective attention [29]. Because of

271 the coarse-grained similarities between video game playing and MIS (e.g., cursor control,

272 complex task environment), it is possible that expert surgeons possess enhanced

273 attentional capabilities, and this is what confers performance benefits in our visuomotor

274 adaptation task. Despite its plausibility, specific features of our paradigm make this 275 explanation unlikely. Even if the surgeons we tested have superior attentional capacities

276 compared to controls, the task is not attentionally demanding since only a single reach

277 target is presented per trial without the appearance of any distractors. Although attention

278 has been shown to effect generalization of visuomotor learning [30, 31], all of these 279 studies use concurrent attention-demanding secondary tasks during the adaptation 280 period which differs substantially from the paradigm we employed.

Another, more probable explanation is that minimally invasive surgeons are exploiting additional resources such as explicit strategies or heuristics to adapt faster and

283 more completely than controls. Although a long-standing view has been that visuomotor 284 adaptation reflects incremental (i.e., trial-by-trial) error-based learning that occurs in an 285 implicit and automatic manner, recent experimental and computational modelling work 286 indicates that it often depends on the operation of multiple interacting learning processes $287[32,33]$. For example, if a perturbation induces a large reaching error to the right side of 
288 the target, subjects experiencing this error signal might consciously choose to aim to the 289 left side of the target on the next trial in order to compensate. One possibility is that our

290 expert surgeons deployed similar strategies and did so more rapidly and effectively adapt

291 than controls. Further studies will be required to tease apart the roles of these different

292 visuomotor learning processes in expert surgeons.

Although visuomotor adaptation has been extensively studied [17, 2], few researchers have looked at learning differences across individuals or between specific groups. To date, there has been limited exploration of individual differences in visuomotor adaptation [34, 35], and very few studies have investigated adaptation differences

297 between experts such as professional athletes and novices [36, 37]. This relative lack of attention may reflect a common background assumption in the field of motor learning that visuomotor adaptation performance should be largely uniform across the adult human population. Moreover, while expertise and expert performance have been thoroughly 301 investigated from both psychological [38,39] and neuroscientific perspectives [40], 302 surprisingly little attention has been given to surgeons as an informative expert cohort.

303 The current study begins to close this important gap by showing that expert minimally 304 invasive surgeons exhibit enhanced visuomotor learning in every measure we tested. 305 Specifically, experts adapted to the imposed visuomotor rotation more rapidly and more 306 completely than controls. They also generalized their learning to new target directions 307 more broadly and to a considerably greater extent. 
311 Similar to our study, they reported lower movement variability (higher consistency) and

312 greater accuracy in their expert group prior to learning, both widely considered to be

313 hallmarks of expert performance [41]. But interestingly, they observed no learning rate

314 differences in one experiment and a slower rate of adaptation in experts compared to

315 novices in another experiment. One plausible explanation for the large discrepancy

316 between their findings and ours is that handball players do not have to contend with or

317 achieve mastery over changes in visuomotor mappings as do expert surgeons with

318 extensive training and experience performing MIS.

Despite its major benefits for patients compared to open surgery, it is now widely

320 recognized that minimally invasive surgery is inherently challenging to learn and can even

321 be prohibitively difficult for some surgical residents such that they never reach proficiency

$322[42,43,44]$. Pinpointing the underlying sources of these learning curve differences remains

323 an unanswered challenge. The findings reported here indicate that differences in

324 visuomotor learning capacities, either innate or acquired, might be an important source

325 of difficulty for learning to perform MIS. This information can be used to help guide surgical

326 candidate selection or optimize training programs to address the specific needs of

327 individual trainees. Our study also demonstrates that a general visuomotor learning

328 paradigm from the field of motor neuroscience can reliably distinguish expert from non-

329 expert in MIS. This result opens the door for the exploration of other common paradigms

330 such as gain adaptation [18], which may shed valuable light on learning and expert

331 performance in increasingly dominant approaches in surgical medicine such as robot-

332 assisted minimally invasive surgery. 


\section{References}

334 1. Shadmehr R, Smith MA, Krakauer JW. Error correction, sensory prediction, and

335 adaptation in motor control. Annu Rev Neurosci. 2010;33:89-108.

336 2. Krakauer JW, Mazzoni P. Human sensorimotor learning: adaptation, skill, and beyond. Curr Opin Neurobiol. 2011 Aug;21(4):636-44.

3. Cuschieri A. Whither minimal access surgery: tribulations and expectations. Am J Surg. 1995 Jan;169(1):9-19.

4. Braga M, Vignali A, Gianotti L, Zuliani W, Radaelli G, Gruarin P, et al. outcome. Ann Surg. 2002 Dec;236(6):759-66; disscussion 767.

5. den Boer KT, de Jong T, Dankelman J, Gouma DJ. Problems with Laparoscopic Instruments: Opinions of Experts. Journal of Laparoendoscopic \& Advanced Surgical Techniques. 2001 Jun;11(3):149-55.

6. Joice P, Hanna GB, Cuschieri A. Ergonomic evaluation of laparoscopic bowel suturing. Am J Surg. 1998 Oct;176(4):373-8.

7. Wentink B. Eye-hand coordination in laparoscopy - an overview of experiments and supporting aids. Minim Invasive Ther Allied Technol. 2001 Jan;10(3):155-62.

350 8. Rattner DW. Beyond the laparoscope: minimally invasive surgery in the new millennium. Surgery. 1999 Jan;125(1):19-22.

9. Schauer P, Ikramuddin S, Hamad G, Gourash W. The learning curve for Feb;17(2):212-5. 
10. Heuer H, Siilzenbrijck S. 2 Tool Use in Action: The Mastery of Complex

Visuomotor. Action science: Foundations of an .... 2013;

11. Prinz W, Beisert M, Herwig A. Action science: foundations of an emerging

12. Pouget A, Snyder LH. Computational approaches to sensorimotor transformations. Nat Neurosci. 2000 Nov;3 Suppl:1192-8.

13. Soechting JF, Flanders M. Errors in pointing are due to approximations in sensorimotor transformations. J Neurophysiol. 1989 Aug;62(2):595-608.

14. Flanders M, Tillery SIH, Soechting JF. Early stages in a sensorimotor transformation. Behav Brain Sci. 1992 Jun;15(02):309-20.

15. Sober SJ, Sabes PN. Flexible strategies for sensory integration during motor planning. Nat Neurosci. 2005 Apr;8(4):490-7.

16. Cunningham HA. Aiming error under transformed spatial mappings suggests a structure for visual-motor maps. J Exp Psychol Hum Percept Perform. 1989 Aug;15(3):493-506.

17. Krakauer JW. Motor learning and consolidation: the case of visuomotor rotation. Adv Exp Med Biol. 2009;629:405-21.

18. Krakauer JW, Pine ZM, Ghilardi MF, Ghez C. Learning of visuomotor transformations for vectorial planning of reaching trajectories. J Neurosci. 2000 Dec 1;20(23):8916-24. 
fundamentals of laparoscopic surgery. Surg Innov. 2007 Jun;14(2):107-12.

377 20. Gozli DG, Bavelier D, Pratt J. The effect of action video game playing on sensorimotor learning: Evidence from a movement tracking task. Hum Mov Sci. 2014 Oct 12;38C:152-62.

21. Lynch J, Aughwane P, Hammond TM. Video games and surgical ability: a literature review. J Surg Educ. 2010 Jun;67(3):184-9.

22. Li L, Chen R, Chen J. Playing action video games improves visuomotor control. Psychol Sci. 2016 Jul 8;27(8):1092-108.

23. Brayanov JB, Press DZ, Smith MA. Motor memory is encoded as a gain-field combination of intrinsic and extrinsic action representations. J Neurosci. 2012 Oct 24;32(43):14951-65.

24. Georgopoulos AP, Kalaska JF, Caminiti R, Massey JT. On the relations between the direction of two-dimensional arm movements and cell discharge in primate motor cortex. J Neurosci. 1982 Nov;2(11):1527-37.

25. Moran DW, Schwartz AB. Motor cortical representation of speed and direction during reaching. J Neurophysiol. 1999 Nov;82(5):2676-92.

26. Scott SH, Gribble PL, Graham KM, Cabel DW. Dissociation between hand motion and population vectors from neural activity in motor cortex. Nature. $2001 \mathrm{Sep}$

27. Taylor JA, Krakauer JW, Ivry RB. Explicit and implicit contributions to learning in a sensorimotor adaptation task. J Neurosci. 2014 Feb 19;34(8):3023-32. 
28. Poggio T, Bizzi E. Generalization in vision and motor control. Nature. 2004 Oct $14 ; 431(7010): 768-74$

29. Green CS, Bavelier D. Action video game modifies visual selective attention.

Nature. 2003 May 29;423(6939):534-7.

30. Bédard P, Song J-H. Attention modulates generalization of visuomotor adaptation.

J Vis. 2013 Oct 16;13(12):12.

31. Wang TSL, Song J-H. Impaired visuomotor generalization by inconsistent attentional contexts. J Neurophysiol. 2017 Sep 1;118(3):1709-19.

32. Mazzoni P, Krakauer JW. An implicit plan overrides an explicit strategy during visuomotor adaptation. J Neurosci. 2006 Apr 5;26(14):3642-5.

33. McDougle SD, Boggess MJ, Crossley MJ, Parvin D, Ivry RB, Taylor JA. Credit assignment in movement-dependent reinforcement learning. Proc Natl Acad Sci USA. 2016 Jun 14;113(24):6797-802.

34. Christou AI, Miall RC, McNab F, Galea JM. Individual differences in explicit and implicit visuomotor learning and working memory capacity. Sci Rep. 2016 Nov

35. Stark-Inbar A, Raza M, Taylor JA, Ivry RB. Individual differences in implicit motor learning: task specificity in sensorimotor adaptation and sequence learning. $\mathrm{J}$ Neurophysiol. 2017 Jan 1;117(1):412-28.

416 36. Leukel C, Gollhofer A, Taube W. In Experts, underlying processes that drive 
37. Kast V, Leukel C. Motor Experts Care about Consistency and Are Reluctant to

Change Motor Outcome. PLoS ONE. 2016 Aug 30;11(8):e0161798.

421

422

423

424

425

426

427

428

429

430

431

432

433

434

435

436

437

438

439

38. Ericsson KA, Lehmann AC. Expert and exceptional performance: evidence of maximal adaptation to task constraints. Annu Rev Psychol. 1996;47:273-305.

39. Ericsson KA, Nandagopal K, Roring RW. Toward a science of exceptional achievement: attaining superior performance through deliberate practice. Ann N Y Acad Sci. 2009 Aug;1172:199-217.

40. Yarrow K, Brown P, Krakauer JW. Inside the brain of an elite athlete: the neural processes that support high achievement in sports. Nat Rev Neurosci. 2009 Aug;10(8):585-96.

41. Willingham DB. A neuropsychological theory of motor skill learning. Psychol Rev. 1998 Jul;105(3):558-84.

42. Grantcharov TP, Funch-Jensen P. Can everyone achieve proficiency with the laparoscopic technique? Learning curve patterns in technical skills acquisition. Am J Surg. 2009 Apr;197(4):447-9.

43. Buckley CE, Kavanagh DO, Gallagher TK, Conroy RM, Traynor OJ, Neary PC. Does aptitude influence the rate at which proficiency is achieved for laparoscopic appendectomy? J Am Coll Surg. 2013 Dec;217(6):1020-7.

44. Buckley CE, Kavanagh DO, Nugent E, Ryan D, Traynor OJ, Neary PC. The impact of aptitude on the learning curve for laparoscopic suturing. Am J Surg. 2014 Feb;207(2):263-70. 


\section{Baseline}

\section{Adaptation}

\section{Generalization}

Target workspace
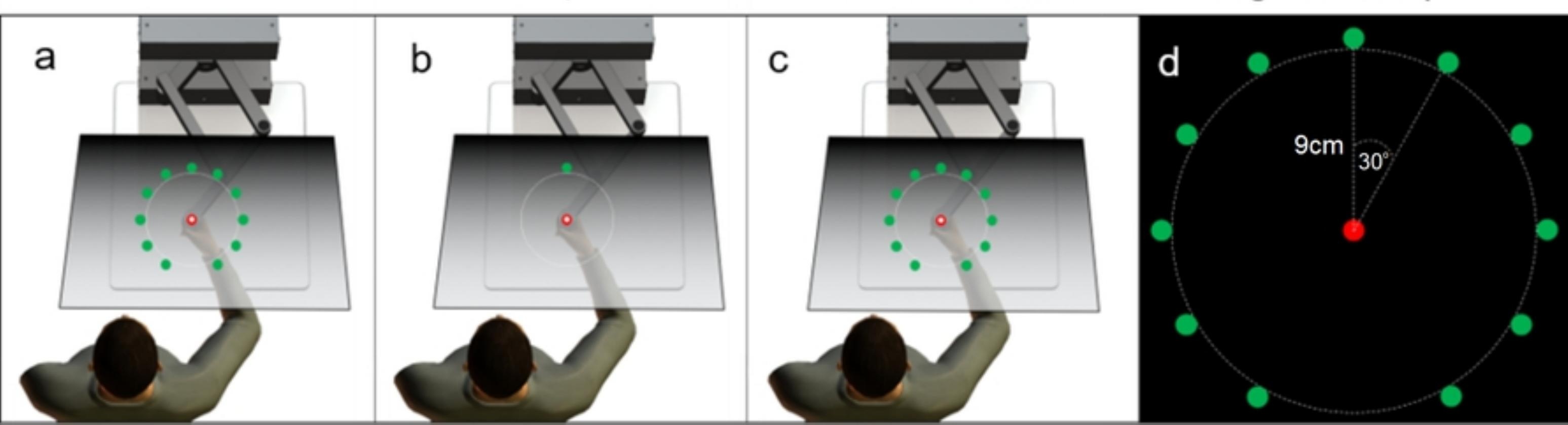


\section{Expert}

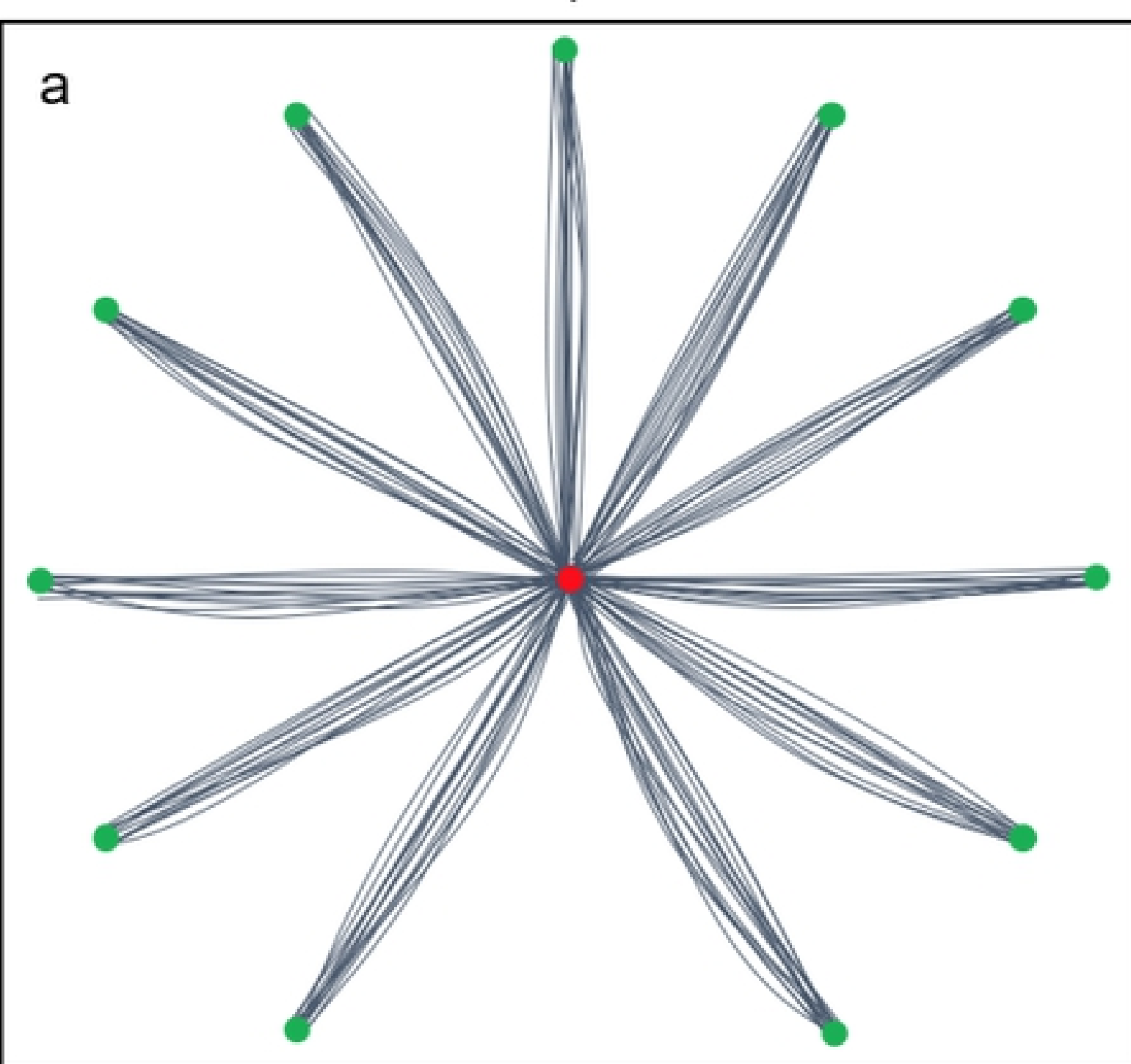

Control

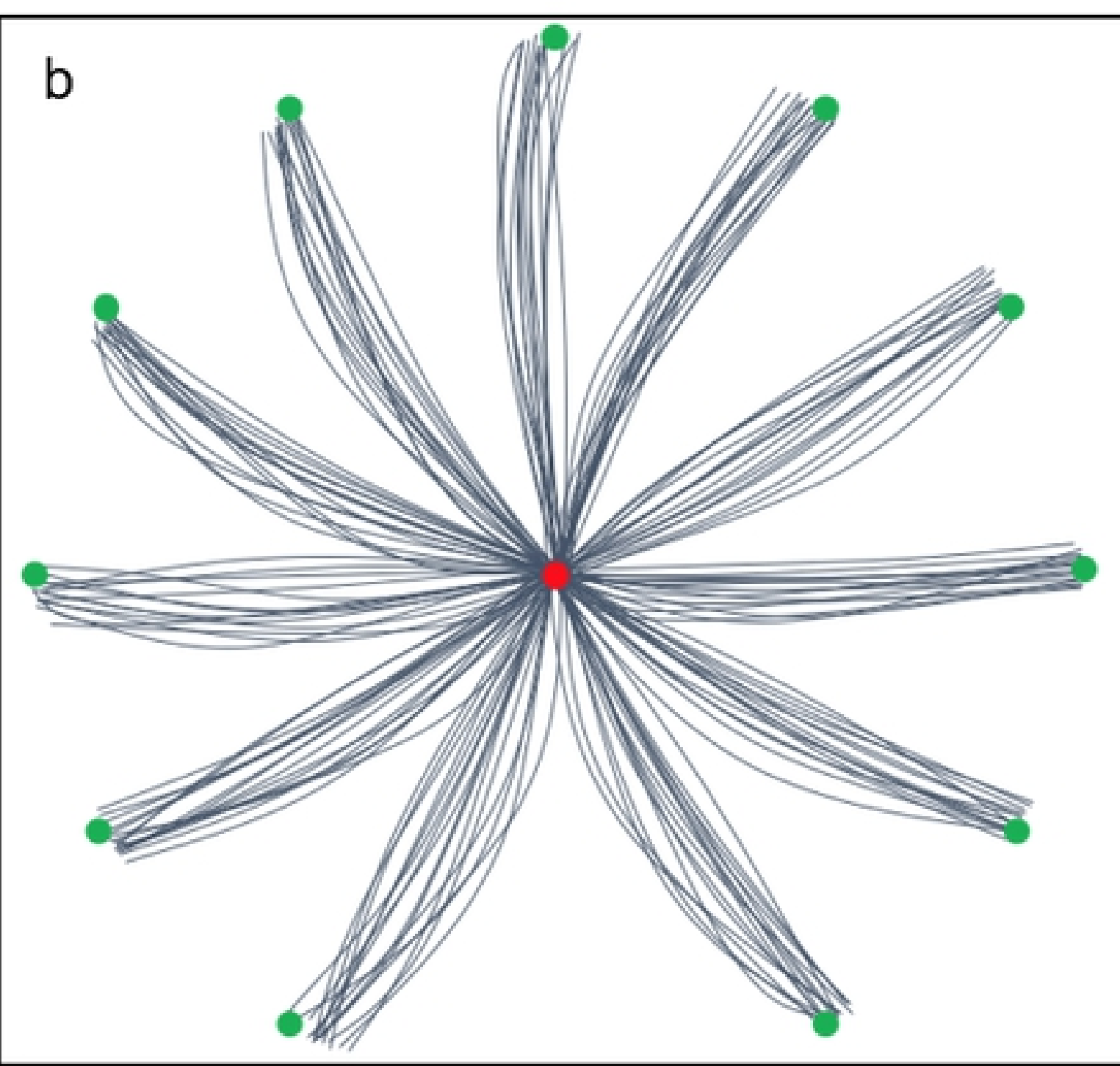




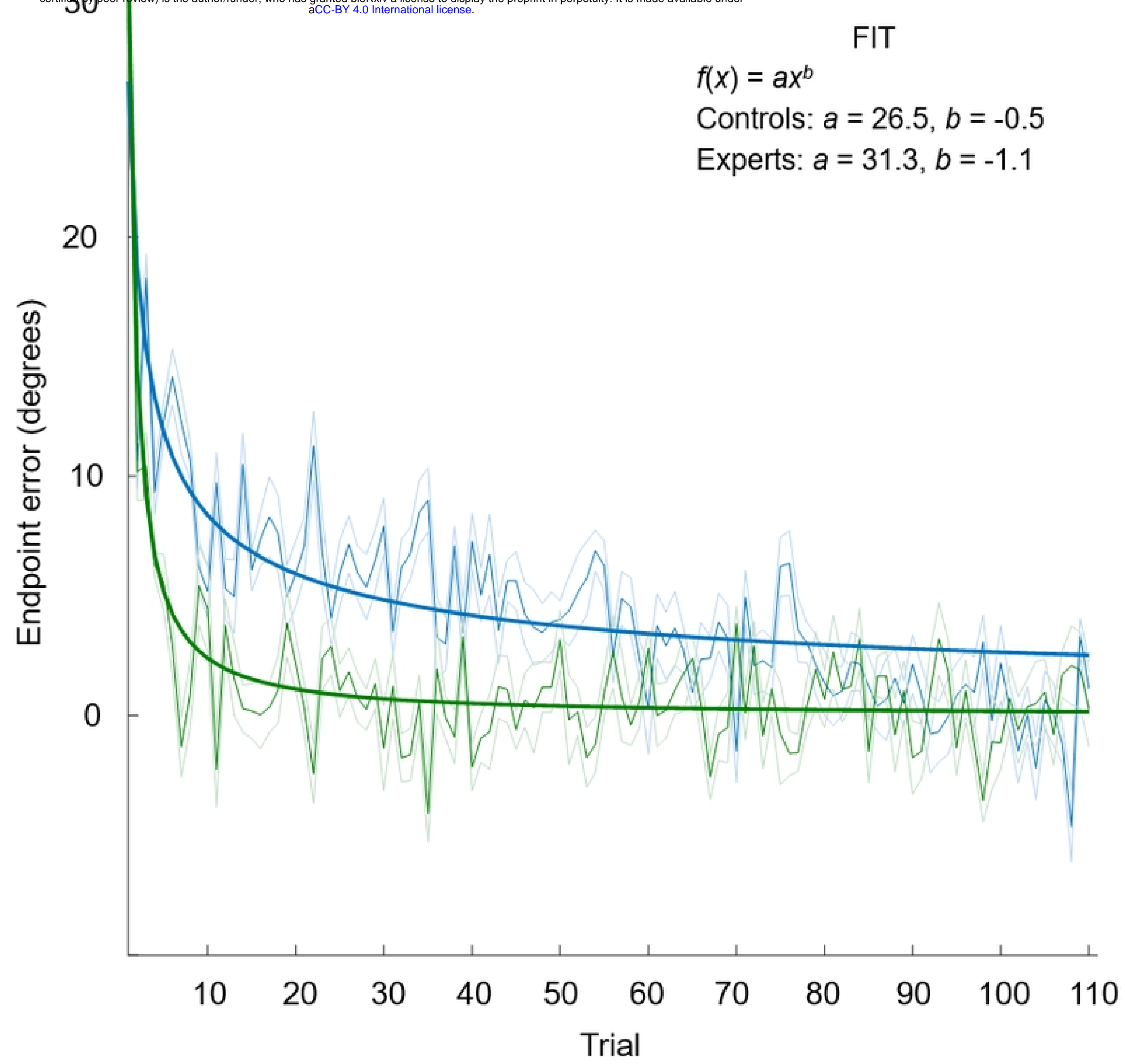




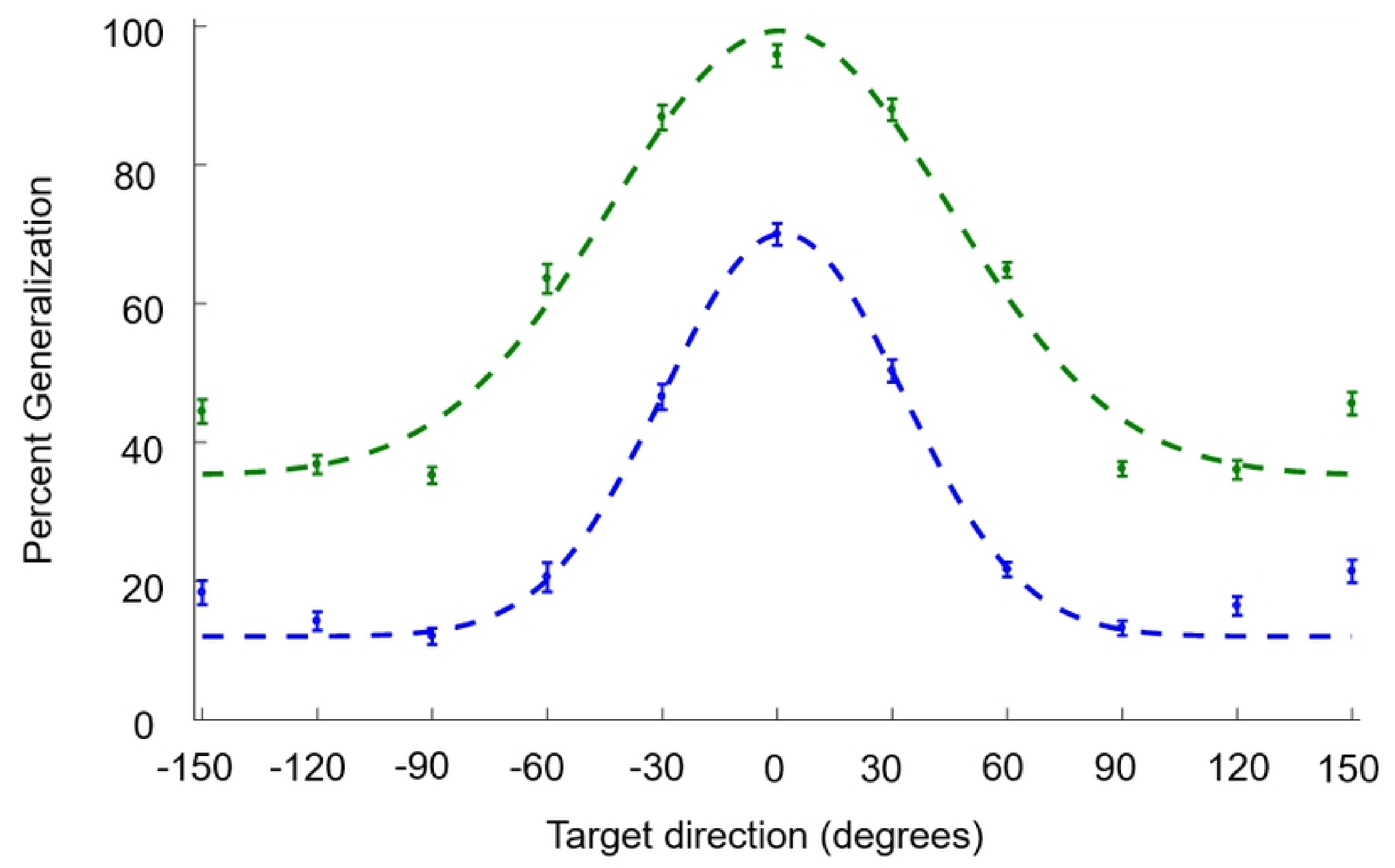

